\title{
Physics of Heliotron J Confinement
}

Fumimichi SANO, Tohru MIZUUCHI, Kazunobu NAGASAKI, Kiyoshi HANATANI,

Hiroyuki OKADA, Yuji NAKAMURA ${ }^{1)}$, Takashi MINAMI, Shinji KOBAYASHI, Satoshi YAMAMOTO, Shigeru KONOSHIMA, Shinsuke OHSHIMA ${ }^{2)}$, Masaki TAKEUCHI, Yoshiyuki IJIRI, Keiji YAGUCHI, Tohru SENJU, Masashi SHIBANO, Kiyoshi TOHSHI, Kinzou SAKAMOTO, Akinobu MATSUYAMA ${ }^{1)}$, Kiyofumi MUKAI ${ }^{1)}$, Keishi MINAMI ${ }^{1)}$,

Shintarou KISHI ${ }^{1)}$, Hyunyong LEE $^{1)}$, Yu TAKABATAKE ${ }^{1)}$, Hiroaki YASHIRO ${ }^{1)}$,

Kento YAMAMOTO $^{1)}$, Kohta NOMURA ${ }^{1)}$, Masashige SUWA $^{1)}$, Hayao YOSHINO ${ }^{1)}$, Sadayoshi MURAKAMI ${ }^{3)}$, Takashi MUTOH ${ }^{4)}$, Yasuhiko TAKEIRI ${ }^{4)}$, Kenichi NAGAOKA ${ }^{4)}$, Shoichi OKAMURA ${ }^{4)}$, Kiyomasa Y. WATANABE ${ }^{4)}$, Masayuki YOKOYAMA ${ }^{4)}$, Yasuhiro SUZUKI ${ }^{4)}$, Yasuo YOSHIMURA ${ }^{4)}$, Shin NISHIMURA ${ }^{4)}$, Naoki TAMURA ${ }^{4)}$, Satoru SAKAKIBARA ${ }^{4)}$, Gen MOTOJIMA ${ }^{4}$, Nobuhiro NISHINO $^{5)}$, Takeshi FUKUDA ${ }^{6}$, Yousuke NAKASHIMA ${ }^{7)}$, Zhen FENG ${ }^{8)}$, Qingwei YANG $^{8)}$, Angela FERNÁNDEZ ${ }^{9)}$, Alvaro CAPPA $^{9)}$, Victor TRIBALDOS ${ }^{9)}$, Boyd D. BLACKWELL ${ }^{10)}$ and Viktor V. CHECHKIN ${ }^{11)}$

Institute of Advanced Energy, Kyoto University, Kyoto 611-0011, Japan

${ }^{1)}$ Graduate School of Energy Science, Kyoto University, Kyoto 611-0011, Japan

${ }^{2)}$ Kyoto University Pioneering Research Unit for Next Generation, Kyoto 611-0011, Japan

${ }^{3)}$ Graduate School of Engineering, Kyoto University, Kyoto 606-8501, Japan

${ }^{4)}$ National Institute for Fusion Science, Gifu 509-5292, Japan

${ }^{5)}$ Graduate School of Engineering, Hiroshima University, Hiroshima 739-8527, Japan

${ }^{6}{ }^{6}$ Graduate School of Engineering, Osaka University, Osaka 565-0871, Japan

${ }^{7)}$ Plasma Research Center, University of Tsukuba, Ibaraki 305-8577, Japan

${ }^{8)}$ Center for Fusion Science, Southwestern Institute of Physics, Sichuan 610041, China

${ }^{9}$ Laboratorio Nacional de Fusion, EURATOM-CIEMAT, 28040 Madrid, Spain

${ }^{10)}$ Australian National University, Canberra, Australia

${ }^{11)}$ Institute of Plasma Physics, NSC KIP, 61108 Kharkov, Ukraine

(Received 7 December 2009 / Accepted 12 April 2010)

This paper reviews the results of an experimental study undertaken in Heliotron $\mathbf{J}$ over the past few years to explore the physics design base for a new concept of a helical-axis heliotron. Measurements of electron cyclotron resonance $(\mathrm{ECR})$ /neutral beam injection (NBI)/ion cyclotron range of frequencies (ICRF) heating plasmas have been made for understanding global energy confinement in connection with the international stellarator scaling law (ISS04), spontaneous confinement improvement (L-H transition), confinement improvement based on supersonic molecular beam injection (SMBI), magnetohydrodynamic (MHD) activity, edge plasma characteristics, including rotation of a filamentary turbulence structure, and plasma current control, including the electron cyclotron current drive (ECCD), the energetic-particle driven Alfvén eigenmodes, and related fast ion dynamics. The results are discussed in terms of the rotational transform $\iota / 2 \pi$ and the bumpiness $\varepsilon_{\mathrm{b}}$ (or the effective helical ripple $\varepsilon_{\text {eff }}$ ). Control of these two parameters was experimentally demonstrated to be the key issue in determining the optimum performance of Heliotron J. The result confirms that the helical-axis heliotron provides a unique and high potential for exploiting an alternative and advanced path to future helical systems.

(C) 2010 The Japan Society of Plasma Science and Nuclear Fusion Research

Keywords: Heliotron J, helical-axis heliotron, bumpiness, stellarator, confinement improvement, L-H transition, H-mode, ISS04, MHD, ECCD, SMBI, Alfvén eigenmode, advanced helical system

DOI: $10.1585 /$ pfr.5.S2003

\section{Introduction}

Heliotron $\mathrm{J}$ is a medium-sized, low magnetic shear, $L$ $=1 / M=4$ helical-axis heliotron $(R=1.2 \mathrm{~m}, a=0.2 \mathrm{~m}, B<$ $1.5 \mathrm{~T}$ ) with an averaged magnetic well; it is equipped with

author'se-mail: sano@iae.kyoto-u.ac.jp several heating systems, such as $0.4 \mathrm{MW}$ ECH, $1.5 \mathrm{MW}$ NBI and $0.5 \mathrm{MW}$ ICRF $[1,2] . R$ is the major radius of the torus, $a$ is the minor radius of the plasma, $B$ is the magnetic field strength, $L$ is the pole number of the helical field coil, and $M$ is the pitch number of the field along the toroidal direction. The plasma operation regimes are widely extended 
by virtue of its configuration flexibility. This feature permits a unique investigation of the currentless helical-axis heliotron and provides a platform for studying relevant theoretical models of confinement. For effective particle confinement in earlier planar-axis heliotrons, such as Heliotron $\mathrm{E}(R=2.2 \mathrm{~m}, a=0.2 \mathrm{~m}, B<2.0 \mathrm{~T})$ [3], drift optimization was realized by an inward magnetic axis shift while the edge magnetic hill region was expanded [4]. The large helical device (LHD) $(R=3.9 \mathrm{~m}, a=0.6 \mathrm{~m}, B<$ $3.0 \mathrm{~T})$ [5] also follows the same principle of drift optimization. The high shear common in such devices provides a stabilizing term for MHD instability. However, in the LHD, energy confinement is considered to degrade at high beta values (volume-averaged beta value $\langle\beta>>2 \%$ ) because the resistive interchange mode is not stabilized by the shear in the edge magnetic hill region $[6,7]$.

In new helical-axis heliotrons such as Heliotron J, drift optimization is achieved by a new control knob, the bumpiness, completely independent of the magnetic axis shift while maintaining the vacuum magnetic well over the entire volume. The magnetic well configuration is one of the key advantages of Heliotron $\mathrm{J}$ in maintaining good confinement at high beta values. In addition, the magnetic well can satisfy the typical Mercier criterion up to $\langle\beta>\sim 4 \%$ [8] in contrast to a value of $\langle\beta\rangle \sim 2 \%$ for Heliotron $\mathrm{E}$. The primary goal of Heliotron $\mathrm{J}$ is to contribute to the physical understanding of configuration optimization of the heliotron line, although a similar low-shear concept with a magnetic well using modular coils was studied in W7-AS $(R=2.0 \mathrm{~m}, a=0.2 \mathrm{~m}, B<3 \mathrm{~T})$ and will be further investigated in W7-X $(R=6.5 \mathrm{~m}, a=0.65 \mathrm{~m}, B<3 \mathrm{~T})$ to develop a modular stellarator line originally based on quasi-helical symmetry [9]. Complementary experiments in Heliotron $\mathrm{J}$ and W7-X will be useful for the better understanding of the different optimization scenarios required for each line. To attain a high degree of compatibility between good particle confinement and MHD stability in Heliotron $\mathrm{J}$, the rotational transform and bumpiness are the key elements of physics research [10]. In this connection, their effects on various aspects of confinement have been investigated experimentally; this paper reviews the results.

\section{Physics Design}

Heliotron $\mathrm{J}$ was constructed as a post-Heliotron E device to explore a new concept of the currentless helicalaxis heliotron toward a steady-state, compact high- $\beta$ fusion reactor [1]. Its physics design is based on the principles of (a) omnigeneity for drift particle confinement and (b) a magnetic well for MHD stability. The earlier Heliotron $\mathrm{E}$ was a high-rotational-transform $[\iota(a) / 2 \pi \sim 2.5]$ and high-shear device with a planar magnetic axis. The aspect ratio $R / a$ was about 11 . The new features of Heliotron $\mathrm{J}$ are (a) medium rotational transform $[\iota(a) / 2 \pi \sim 0.3-0.8]$, (b) low shear, and (c) a magnetic well with a helical magnetic axis. Its more compact aspect ratio $R / a$ is about 6 to 7. Its plasma column spirals around a helical field coil buried in the torus surface, creating an averaged magnetic well over the entire volume that increases in depth with increase in minor radius up to $\delta_{\mathrm{w}}(a) \sim 2 \%$, which is in marked contrast to the edge magnetic hill of Heliotron E. Here $\delta_{\mathrm{w}}(a)$ is the well depth at the plasma boundary. The rotational transform of Heliotron $\mathrm{J}$ is created by the external coils of the negatively pitch-modulated helical field coil (pitch-modulation factor $\alpha=-0.4$ ) and the toroidal field coils. The latter are composed of two types of coils (type A and type B), which are also used to control the toroidal mirror field, i.e., the bumpiness. Since Heliotron J is a lowshear device, the standard profile of the vacuum rotational transform $\left[\iota(a) /\left.2 \pi\right|_{\text {vac }} \sim 0.56\right]$ avoids low-order rationals entirely. However, the existence of its finite shear is expected to positively affect confinement. The magnetic axis is controlled by two kinds of vertical field coils (AV and IV coils). This simple coil system is an obvious advantage of Heliotron $\mathrm{J}$, and it offers operational flexibility that is needed for concept exploration studies.

The drift optimization applied to Heliotron $\mathbf{J}$ aims to reduce the field harmonics of the magnetic configuration in the Boozer coordinates to only a few dominant harmonics such as helicity $\left(\varepsilon_{\mathrm{h}}\right)$, toroidicity $\left(\varepsilon_{\mathrm{t}}\right)$, and bumpiness $\left(\varepsilon_{\mathrm{b}}\right)$ to acquire a good vacuum configuration without large islands in the core region and with a smooth separatrix in the boundary region and to reduce cross-field transport. As a design criterion, if Heliotron $\mathrm{J}$ follows the reactor scenario in which the core configuration is maintained as much as possible throughout the plasma evolution, the edge field topology should also be robust to achieve reliable divertor operation. The divertor of a helical-axis heliotron is assembled using large islands in the scrape-off-layer (SOL) region; therefore, the boundary rotational transform should also be kept constant to maintain divertor capability. Thus the currentless operation based on the optimized vacuum configuration is one of the attractive routes for the development of the helical-axis heliotron reactor. The currentless scenario also leads to the reduction of the free energy to excite current-driven instabilities and thus contributes to disruption-free operation. To exploit the distinct advantage of the currentless scenario, bootstrap current minimization is an advantageous design criterion. In fact, the bootstrap current should be small enough to afford a good margin for net current reduction using physics tools such as ECCD or neutral beam current drive (NBCD). As a consequence, a high degree of compatibility between good particle confinement and MHD stability under currentless conditions is the immediate objective of physics research on Heliotron $\mathbf{J}$ confinement.

In quasi-symmetry devices such as HSX [11], good particle confinement is realized by substantial reduction of ripple-trapped particles, but these designs impose a severe constraint on the design parameter space, and the freedom to effectively control the bootstrap current as well as other aspects of confinement is lost. On the other hand, the de- 
signs that achieve omnigeneity by drift optimization, such as the Heliotron $\mathbf{J}$ design, can provide potentially greater freedom to control the bootstrap current. Bootstrap current minimization can be achieved in Heliotron $\mathrm{J}$ by the intrinsic interaction between the non-zero $\varepsilon_{\mathrm{t}}$ and $\varepsilon_{\mathrm{h}}$ components and a properly chosen $\varepsilon_{\mathrm{b}}$ component.

In terms of thermal confinement under the existence of turbulent transport, the drift optimization of Heliotron $\mathbf{J}$ presents a reasonably low level of the effective helical ripple, $\varepsilon_{\text {eff }}$ (the figure of merit for neoclassical optimization) [12], that is less than that of Heliotron E, within the constraints of a simple coil system using a continuous helical field coil to provide enough experimental flexibility [13]. In the drift optimization of Heliotron $\mathrm{J}$, even a small radial electric field $E_{\mathrm{r}}$ in the electron/ion root regimes can also improve the orbit confinement of ripple-trapped particles due to their low $\nabla B$-drift velocities in the omnigeneous configuration [14].

Plasma equilibrium is realized to such an extent that $\beta$ self-stabilization is expected with increasing well depth at high $\beta$ values together with a controlled inward magnetic axis shift that counteracts the Shafranov shift. In this case, to maintain the magnetic configuration without large islands and stochastic regions is the main focus of the research. In addition, $\beta$ self-improvement of drift optimization is expected due to the configuration shift toward poloidal symmetry at high $\beta$ values. These high- $\beta$ issues remain to be addressed in an integrated optimization study of a helical-axis heliotron.

\section{Experimental Results and Discus- sion}

\subsection{Thermal confinement}

\subsubsection{International stellarator scaling law (ISS04)}

The global energy confinement characteristics of the second harmonic $70 \mathrm{GHz} \mathrm{ECH}$ plasmas at $B=1.0-1.5 \mathrm{~T}$ in the standard configuration have been studied with special reference to collaborative studies of the international stellarator confinement database [15]. The results show that the data from Heliotron $\mathrm{J}$ are broadly comparable to those of other helical systems such as the LHD, W7-AS, and TJ-II, and that they follow the general tendencies of the unified scaling law ISS04 with a renormalization factor $f=0.6$. The ISS04 scaling shows properties of the gyro-Bohm type, and the dimensional characteristics are roughly consistent with the fluctuation-induced transport of the collisional high- $\beta$ model. A similarity in the rotational transform dependence $[\iota(2 a / 3) / 2 \pi]^{04}$ also exists between the ISS04 scaling and the Lackner-Gottardi scaling [16], where the global confinement improves with increase in the rotational transform. The renormalization factor $f$ is interpreted to represent the addition of the specific configuration effects to the iota dependence for each device; this database suggests a statistical improvement in confinement with a reduction of $\varepsilon_{\text {eff }}$ [15], as shown in Fig. 1. This find-

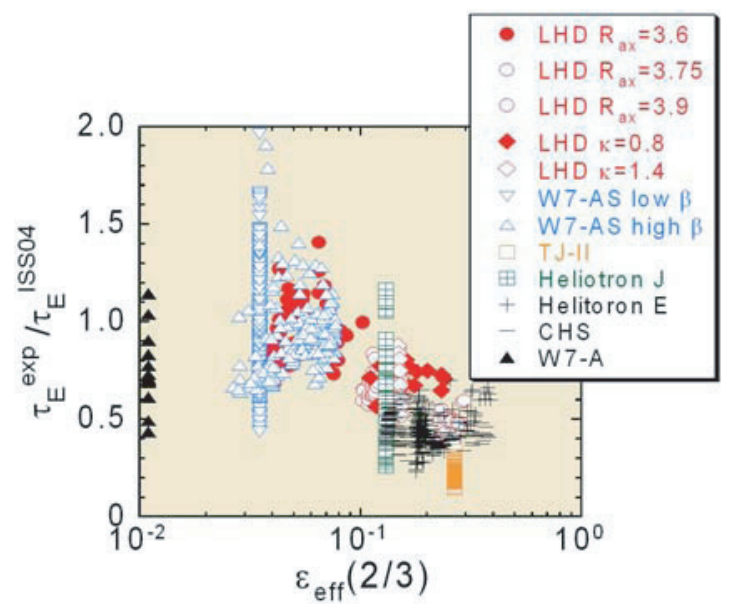

Fig. 1 Effective helical ripple $\left(\varepsilon_{\text {eff }}\right)$ dependence of $\tau_{\mathrm{E}}^{\exp } / \tau_{\mathrm{E}}^{\mathrm{ISS} 04}$ for ISS04 data.

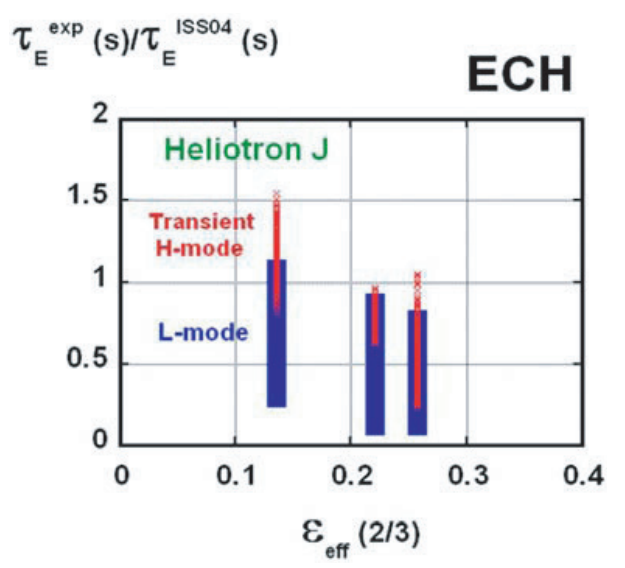

Fig. 2 Effective helical ripple ( $\varepsilon_{\text {eff }}$ ) dependence of $\tau_{\mathrm{E}}^{\exp } / \tau_{\mathrm{E}}^{\mathrm{ISS} 04}$ for Heliotron J ECH plasmas with bumpiness-modified configurations [8].

ing was also confirmed qualitatively with $\mathrm{ECH}$ plasmas (Land $\mathrm{H}$-mode together) in Heliotron $\mathrm{J}$, although the $\varepsilon_{\text {eff }}$ scan range was limited [13], as shown in Fig. 2. This raises an important issue: neoclassical optimization can reduce the turbulent transport that governs the outer confinement region. A model of the relevant physics has been proposed in Ref. [17].

\subsubsection{H-mode}

The L-H transition of Heliotron $\mathrm{J}$ has been studied with special regard to its characteristics compared to those of tokamaks or other helical systems. Spontaneous increases in both electron density $\left(n_{\mathrm{e}}\right)$ and internal plasma energy $\left(W_{\mathrm{p}}\right)$, accompanied by a drop in $\mathrm{H}_{\alpha} / \mathrm{D}_{\alpha}$ emission, are the signature of the $\mathrm{L}-\mathrm{H}$ transition. The $\mathrm{H}$-mode quality for $\mathrm{ECH}+\mathrm{NBI}$ plasmas was examined with an emphasis on its edge rotational transform dependence $[18,19]$. First, the vacuum edge rotational transform $\iota(a) /\left.2 \pi\right|_{\text {vac }}$ was chosen as a label to identify the configuration, although the possible influences of the finite beta and the plasma cur- 
rent should not be neglected in detailed discussions. The VMEC equilibrium calculation predicted that the change in $\iota(a) / 2 \pi$ at the volume-averaged beta value $<\beta><0.3 \%$ is $<(-) 0.020$. The change in $\iota(a) / 2 \pi$ at plasma currents $<2$ $\mathrm{kA}$ is $<(+) 0.015$. Under the given experimental condition, these two effects were compensatory [20]. The experimental $\iota(a) /\left.2 \pi\right|_{\text {vac }}$ dependence of the peak enhancement factor of $H_{\text {ISS04 }}\left(=\tau_{\mathrm{E}}^{\exp } / f \times \tau_{\mathrm{E}}^{\mathrm{ISS} 04}\right)$ over the L-mode confinement revealed that specific $\iota(a) /\left.2 \pi\right|_{\text {vac }}$ windows exist in which high-quality $\mathrm{H}$-modes $\left(H_{\mathrm{ISS} 04}>1.5\right)$ are attained, where $\tau_{\mathrm{E}}^{\exp }$ is the experimental energy confinement time estimated under the calculated net power absorption. The $\iota(a) /\left.2 \pi\right|_{\text {vac }}$ ranges for these windows are near values that are slightly lower than the major natural resonances. The threshold density of the H-mode, which depends on the rational surface and the heating scenario, is in the region of 0.7-2.0 $\times 10^{19} \mathrm{~m}^{-3}$, e.g., in ECH $(0.29 \mathrm{MW})+\mathrm{NBI}(0.57 \mathrm{MW})$ plasma. Langmuir probe measurements showed a large reduction in fluctuation-induced transport near the last closed flux surface (LCFS), as shown in Fig. 3. In addition, a negative radial electric field $E_{\mathrm{r}}$ (or $E_{\mathrm{r}}$-shear) formed simultaneously near the LCFS at the transition [18]. Furthermore, measurements of the edge turbulence dynamics in ECH plasmas with fast cameras revealed a filamentary turbulence structure along the field lines [21]. The change in the rotational direction of this filamentary structure at the L-H transition from counterclockwise to clockwise suggested the buildup of negative $E_{\mathrm{r}}$ in H-mode. To explain the transition, it is proposed that the plasma turbulence is stabilized by the radial shear of the poloidal plasma flow driven by $\boldsymbol{E} \times \boldsymbol{B}$ forces or by the Reynolds stress associated with the rational surfaces. Measurements by a directional probe revealed the excitation of toroidal plasma flow with its spatial structure through the development of the L$\mathrm{H}$ transition. This flow was found to be damped down at the back transition. With regard to electrostatic fluctuation, the change in the probability density function (PDF) for the fluctuation-induced particle flux outside the LCFS (SOL) has been measured [22]. The result indicated that this PDF has a highly positive tail (outward) in the L-mode, and that this tail direction is reversed (inward) in the H-mode with rapid suppression of the particle flux.

\subsubsection{Bumpiness control of thermal confinement}

The role of the bumpiness is to align the $\bmod -B_{\min }$ contours with the magnetic surfaces [10]. The existence of the closed mod- $B_{\min }$ contours necessitates a certain range of the ratio $\varepsilon_{\mathrm{b}} / \varepsilon_{\mathrm{h}}$, which depends on the $B_{\min }$ value or the particle energy. The ratio $\varepsilon_{\mathrm{b}} / \varepsilon_{\mathrm{h}}$ must be more negative as the ratio $\varepsilon_{\mathrm{t}} / \varepsilon_{\mathrm{h}}$ increases, in order to align the bottom of the magnetic field ripple. Here we define $\varepsilon_{\mathrm{t}}$ as $\varepsilon_{\mathrm{t}}=B_{10} / B_{00}$, $\varepsilon_{\mathrm{h}}$ as $\varepsilon_{\mathrm{h}}=B_{14} / B_{00}$, and $\varepsilon_{\mathrm{b}}$ as $\varepsilon_{\mathrm{b}}=B_{04} / B_{00}$, where $B_{\mathrm{mn}}$ is the Fourier component of $B$ in Boozer coordinates. To extract the key physical aspects for achieving drift optimization, three magnetic configurations were studied: (1) $\varepsilon_{\mathrm{b}}=$ 0.01 (low bumpiness, $\varepsilon_{\text {eff }}=0.26$ ), (2) $\varepsilon_{\mathrm{b}}=0.06$ (medium

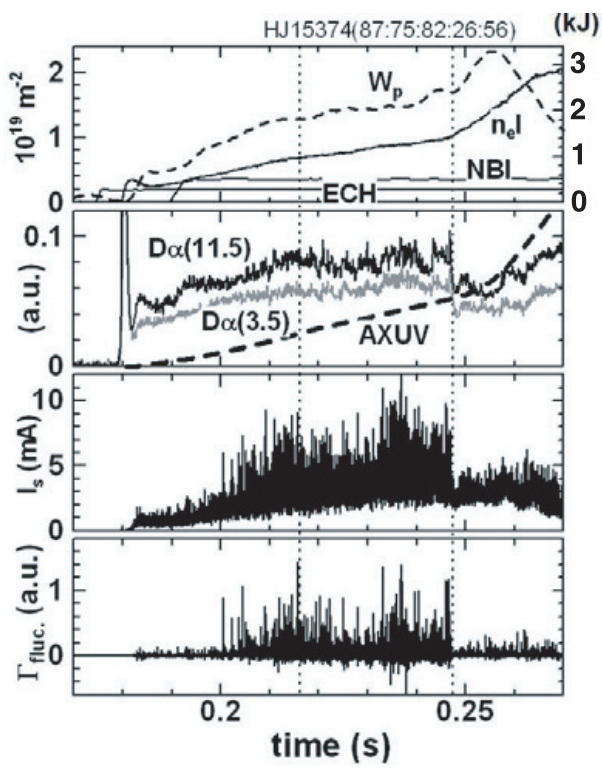

Fig. 3 Time evolution of $\bar{n}_{\mathrm{e}} l, W_{\mathrm{p}}, \mathrm{D}_{\alpha}$ signals (at the port section \#3.5 and \#11.5), AXUV diode signal, Langmuir probe signal $I_{\mathrm{s}}$ and the fluctuation- induced particle flux $\Gamma_{\text {fluc }}$. for $\mathrm{ECH}+\mathrm{NBI}$ plasma. The full transition into $\mathrm{H}$-mode is shown at $t=0.248 \mathrm{~s}$ [18].

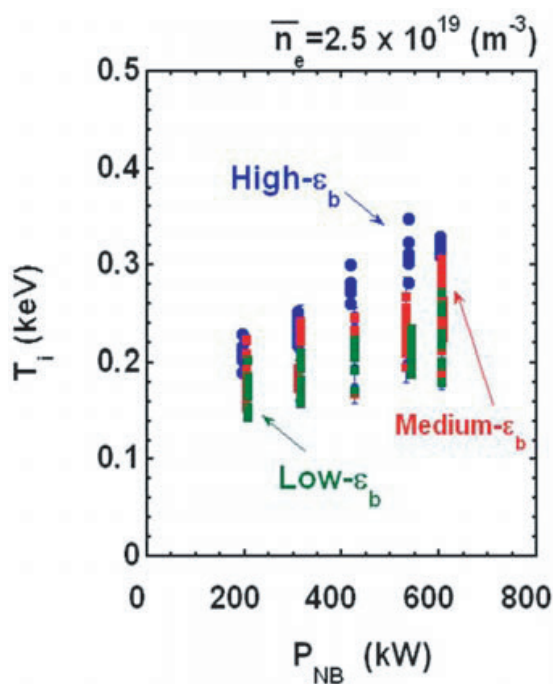

Fig. 4 Ion temperature increase as a function of NBI power for different bumpiness configurations [13].

bumpiness, $\varepsilon_{\text {eff }}=0.13$ ), and (3) $\varepsilon_{\mathrm{b}}=0.15$ (high bumpiness, $\varepsilon_{\text {eff }}=0.22$ ) under similar magnetic axis position, plasma minor radius, and edge rotational transform $\left[\iota(a) /\left.2 \pi\right|_{\mathrm{vac}} \sim\right.$ $0.56]$ conditions, where the values of the bumpiness were chosen at the $2 a / 3$ radius [13]. The volume-averaged internal energy $W_{\mathrm{p}} / V_{\mathrm{p}}$ was chosen as the figure of merit of the thermal confinement under fixed heating conditions, where $V_{\mathrm{p}}$ is the plasma volume. NBI experiments demonstrated that the high- $\varepsilon_{\mathrm{b}}$ configuration provides the best performance, followed by the medium- $\varepsilon_{\mathrm{b}}$ and low- $\varepsilon_{\mathrm{b}}$ configurations. Figure 4 also compares the NBI ion heating effi- 
ciency for the three bumpiness values under the same target density conditions, showing that the high- $\varepsilon_{\mathrm{b}}$ configuration is most efficient. On the other hand, for ECH plasmas, the medium- $\varepsilon_{\mathrm{b}}$ configuration provides the best performance of $W_{\mathrm{p}} / V_{\mathrm{p}}$, followed by the high- $\varepsilon_{\mathrm{b}}$ and low- $\varepsilon_{\mathrm{b}}$ configurations. Further studies will be necessary to understand the source of this difference. For ECH plasmas, as already shown in Fig. 2, the observed order of performance appears to be consistent with the paradigm that the reduction of $\varepsilon_{\text {eff }}$ positively affects turbulent transport, since the medium- $\varepsilon_{\mathrm{b}}$ configuration reduces $\varepsilon_{\text {eff }}$ in the $1 / v$ regime more than the high- $\varepsilon_{\mathrm{b}}$ configuration does.

\subsubsection{Supersonic molecular beam injection (SMBI)}

SMBI was successfully applied to ECH/NBI plasmas [23], showing unique characteristics such as an increase (or decrease) in electron temperature and its target density dependence for ECH plasmas. For ECH (0.35 MW) $+\mathrm{NBI}(0.6 \mathrm{MW})$ plasmas, the stored energy reached about $4.5 \mathrm{~kJ}$, which is about $50 \%$ higher than the maximum value achieved so far under conventional gas-puffing control. To encourage this evident improvement in confinement, the operational optimization of SMBI is in progress.

\subsection{High-energy particle confinement}

Using ICRF minority heating [24], fast ion formation and confinement were investigated under a low density of $0.4 \times 10^{19} \mathrm{~m}^{-3}$ with regard to trapped particle control. The fast ion flux (up to $30 \mathrm{keV}$ ) measured by the chargeexchange neutral particle analyzer (CX-NPA) was greatest in the high- $\varepsilon_{\mathrm{b}}$ configuration at an ICRF power of 200$300 \mathrm{~kW}$. Thus, the high- $\varepsilon_{\mathrm{b}}$ configuration was the most favorable for fast ion formation and confinement. Model calculations based on the Monte Carlo method were in general agreement with the experimental data, indicating the existence of a loss region around the perpendicular direction.

The effect of bumpiness on the energetic ion confinement in NBI plasmas [25] was investigated with regard to passing particle control. The $1 / \mathrm{e}$ decay time of the highenergy CX flux after the NB was turned off was found to increase with the bumpiness.

\subsection{MHD activity}

In the standard operation of Heliotron $\mathrm{J}$ at $\iota(a) / 2 \pi$ $\left.\right|_{\text {vac }} \sim 0.56$, the vacuum magnetic well allows the plasmas to remain very quiescent up to the present available beta values $\langle\beta\rangle<0.5 \%$ [26]. The low-frequency magnetic fluctuations $(<10 \mathrm{kHz})$ of the Mirnov coils set on the inside of the vessel wall are characterized by low-amplitude, low-coherence signals and seem to have no direct adverse effect on confinement. Under these conditions, the confinement properties at high beta values are naturally expected to avoid the degradation caused by the resistive interchange mode, since the magnetic well should contribute effectively toward stabilizing that mode. Next-step experi- ments at high beta values are expected to confirm this.

Coherent modes such as $m=2 / n=1, m=5 / n=$ 3 modes and others, however, were found to grow only in specific configurations whose rotational transforms approach the low-order rationals such as $\iota(a) /\left.2 \pi\right|_{\text {vac }} \sim 0.50$ and $\sim 0.60$. For $\iota(a) /\left.2 \pi\right|_{\text {vac }} \sim 0.50$, the $m=2 / n=1$ mode amplitude grew with increase in $\langle\beta\rangle$, indicating pressuredriven interchange instabilities. The interplay between the coherent modes and the rational surface is the subject of ongoing research and may also be important in understanding the preconditions of the H-mode.

In NBI plasmas, energetic-ion-driven Alfvén eigenmodes $(>20 \mathrm{kHz})$ were also observed. In particular, in the high- $\varepsilon_{\mathrm{b}}$ configuration, the eigenmodes became strong with frequency-chirped bursts $(70 \mathrm{kHz}$ to $40 \mathrm{kHz}$ ) that were correlated with the fast ion dynamics. The co-directed ion current of the directional probe used to detect the fast ion flux in the boundary region provided an immediate response to the bursts, but the ctr-directed current provided almost no response [27]. A possible explanation for these responses is a resonant convective oscillation related to fast ion transport.

\subsection{Plasma current control}

The SPBSC neoclassical bootstrap current code [28] predicts that the direction of the bootstrap current will reverse with the control of the inner vertical field, i.e., with a decrease in $\varepsilon_{\mathrm{b}}$. This prediction was verified in perpendicular ECH experiments, in which the EC-driven current could be neglected [29]. Thus, bootstrap current minimization was achieved by bumpiness control, at least in lowdensity ECH plasmas.

ECCD experiments were performed, focusing on the effects of the magnetic field ripple [30,31]. The EC-driven current decreased as power was deposited at the deeper ripple bottom. Reversal of the current direction was observed as the power was deposited at the ripple bottom, indicating that the amplitude and direction of the EC current is determined by the balance between the Fisch-Boozer effect [32] and the Ohkawa effect [33]. The measurement results and the calculations of electron loss in velocity space indicate that the reduction in EC-driven current is related to the generation and confinement of trapped particles. The low efficiency of ECCD compared to that of tokamaks may be due to the strong Ohkawa effect enhanced by toroidal and helical ripples.

Both these experiments demonstrate that the bumpiness effectively controls the trapping of fast electrons as well as the current drive mechanism to realize currentless operation. Net current-free operation of ECH plasma was demonstrated by bootstrap current plus ECCD control.

\section{Summary}

To gain a better understanding of the omnigeneous configuration obtained by drift optimization of Heliotron 
$\mathbf{J}$ was the essential objective of the present research. The key factors responsible for performance improvement are the rotational transform and the bumpiness. Elucidation of the possible cooperative physics coupling between the neoclassical optimization and the turbulent improvement was found to be important with special reference to bumpiness (or $\varepsilon_{\text {eff }}$ ) control. Bumpiness control was also found to be essential for high-energy particle confinement and plasma current control. The H-mode was observed in certain rotational transform windows, which correspond to the vacuum configurations that come close to the low-order rationals. For MHD studies, a much higher $\beta$ regime is desirable for exploring the beneficial effects of the built-in magnetic well. The physical processes involved in profile control, e.g., using SMBI, in the high- $\beta$ or high-pressure regime will be a major focus of research in confinement improvement in the next-step experiments. Preparation for this work is in progress.

\section{Acknowledgments}

The authors are grateful to all the national and international collaboration groups of Heliotron $\mathbf{J}$ for their valuable discussion and continuing encouragement during this study. This study was conducted with the support and under the auspices of the Collaboration Program of the Laboratory for Complex Energy Processes, IAE, Kyoto University, and the NIFS Collaborative Research Program (NIFS04KUHL005, -002, -006-7, -011-12, -01419, -021, -023-29).

[1] F. Sano et al., J. Plasma Fusion Res. SERIES 3, 26 (2000).

[2] T. Obiki et al., Nucl. Fusion 41, 833 (2001).

[3] K. Uo et al., Nuclear Fusion Supplement, 9th International Conference on Plasma Physics and Controlled Nuclear Fusion Research (1982) 2, IAEA-CN-41/L-3, 209 (1983).
[4] T. Obiki et al., Nuclear Fusion Supplement, 13th International Conference on Plasma Physics and Controlled Nuclear Fusion Research (1990) 2, IAEA-CN-53/C-1-1, 425 (1991).

[5] O. Motojima et al., Nucl. Fusion 40, 599 (2000).

[6] K. Y. Watanabe et al., Nucl. Fusion 45, 1247 (2005).

[7] H. Funaba et al., Fusion Sci. Tech. 51, 129 (2007).

[8] M. Wakatani et al., Nucl. Fusion 40, 569 (2000).

[9] C. Beidler et al., Fusion Tech. 17, 148 (1990).

[10] M. Yokoyama et al., Nucl. Fusion 40, 261 (2000).

[11] J. M. Canik et al., Phys. Rev. Lett. 98, 085002 (2007).

[12] C. Beidler et al., Plasma Phys. Control. Fusion 36, 317 (1994).

[13] F. Sano et al., Fusion Energy 2006, Proc. 21 st IAEA Conf. (Chengdu, 2006), IAEA-CN-149/EX/5-5Ra.

[14] T. Obiki et al., Plasma Phys. Control. Fusion 42, 1151 (2000).

[15] H. Yamada et al., Nucl. Fusion 45, 1684 (2005).

[16] K. Lackner et al., Nucl. Fusion 30, 767 (1990).

[17] H. Sugama et al., Phys. Rev. Lett. 94, 115001 (2005).

[18] F. Sano et al., Nucl. Fusion 45, 1557 (2005).

[19] E. Ascasibar et al., Plasma Fusion Res. 3, S1004 (2008).

[20] F. Sano et al., J. Plasma Fusion Res. 79, 111 (2003).

[21] N. Nishino et al., Plasma Fusion Res. 2, S1055 (2007).

[22] S. Watanabe et al., Plasma Fusion Res. 2, S1059 (2007).

[23] T. Mizuuchi et al., 18th Intern. Toki Conf. (Toki, Japan, 2008), P2-16.

[24] H. Okada et al., 22nd IAEA Fusion Energy Conf. (Geneva, Switzerland, 2008), EX/P6-28.

[25] S. Kobayashi et al., 20nd IAEA Fusion Energy Conf. (Vilamoura, Portugal, 2004), EX/P4-41.

[26] S. Yamamoto et al., Fusion Sci. Tech. 51, 92 (2007).

[27] K. Nagaoka et al., Intern. Conf. Plasma Phys. 2008 (Fukuoka, Japan, 2008), P2-156.

[28] K. Y. Watanabe et al., Nucl. Fusion 35, 335 (1995).

[29] F. Sano et al., Fusion Sci. Tech. 46, 288 (2004).

[30] K. Nagasaki et al., Nucl. Fusion 50, 025003 (2010).

[31] G. Motojima et al., Nucl. Fusion 47, 1045 (2007).

[32] N.J. Fisch and A. Boozer, Phys. Rev. Lett. 45, 720 (1980).

[33] T. Ohkawa, General Atomics Report GA-A 13847 (1976). 